\title{
Unconjugated bilirubin ameliorates the inflammation and digestive protease increase in TNBS-induced colitis
}

\author{
JIN-AN ZHOU ${ }^{1}$, MINGSHAN JIANG ${ }^{2 *}$, XINGUANG YANG ${ }^{3}$, YUANLI LIU ${ }^{1}$, JUNYU GUO $^{1}$, \\ JIADONG ZHENG ${ }^{1}$, YILIN QU ${ }^{1}$, YU SONG ${ }^{1}$, RONGYAN LI $^{1}$, XIAOFA QIN $^{4}$ and XIUHONG WANG ${ }^{1 *}$ \\ ${ }^{1}$ Department of Biochemistry and Molecular Biology, Heilongiiang Provincial Science and \\ Technology Innovation Team in Higher Education Institutes for Infection and Immunity, Harbin Medical University; \\ ${ }^{2}$ Department of General Surgery, The Second Affiliated Hospital of Harbin Medical University, Harbin, Heilongjiang 150086; \\ ${ }^{3}$ Department of Biochemistry and Molecular Biology, Daqing Branch of Harbin Medical University, Daqing, \\ Heilongjiang 163319, P.R. China; ${ }^{4}$ GI Biopharma Inc., Westfield, NJ 07090, USA
}

Received November 29, 2016; Accepted May 31, 2017

DOI: $10.3892 / \mathrm{mmr} .2017 .6825$

\begin{abstract}
The authors previously demonstrated that unconjugated bilirubin (UCB) may inhibit the activities of various digestive proteases, including trypsin and chymotrypsin. The digestive proteases in the lower gut are important in the pathogenesis of inflammatory bowel diseases. The effects of UCB on the inflammation and levels of digestive proteases in feces of rats with colitis have not yet been revealed. The present study investigated the effect of UCB on the inflammatory status and levels of trypsin and chymotrypsin in the feces of rats with trinitrobenzenesulfonic acid (TNBS)-induced colitis. The data indicated that treatment with TNBS resulted in a marked reduction in weight gain, which was significantly alleviated in UCB-treated rats. Furthermore, UCB treatment alleviated the inflammation induced by TNBS, detected via macroscopic damage and microscopic inflammation scores, and pro-inflammatory markers including myeloperoxidase (MPO), tumor necrosis factor (TNF)- $\alpha$ and interleukin (IL)-1 $\beta$. Furthermore, rats with colitis demonstrated significant increases in fecal trypsin and chymotrypsin levels, whereas UCB treatment significantly
\end{abstract}

Correspondence to: Professor Mingshan Jiang, Department of General Surgery, The Second Affiliated Hospital of Harbin Medical University, 148 Baojian Road, Nangang, Harbin, Heilongjiang 150086, P.R. China

E-mail: jmsh5031@aliyun.com

Professor Xiuhong Wang, Department of Biochemistry and Molecular Biology, Heilongjiang Provincial Science and Technology Innovation Team in Higher Education Institutes for Infection and Immunity, Harbin Medical University, 194 Xuefu Road, Nangang, Harbin, Heilongjiang 150086, P.R. China

E-mail: wangxh700417@aliyun.com

${ }^{*}$ Contributed equally

Key words: unconjugated bilirubin, TNBS-induced colitis, trypsin, chymotrypsin alleviated these increases. A significant positive correlation was additionally revealed among the pro-inflammatory markers (MPO, TNF- $\alpha$ and IL-1 $\beta$ ) and fecal digestive proteases (trypsin and chymotrypsin) in colitis. The results of the present study demonstrated that UCB ameliorated the inflammation and digestive protease increase in TNBS-induced colitis.

\section{Introduction}

Inflammatory bowel disease (IBD) is a group of immunologically and genetically mediated chronic inflammatory conditions of gastrointestinal (GI) tract, including ulcerative colitis (UC) and Crohn's disease (CD) $(1,2)$. The incidence of IBD was emerged and dramatically increased in the last century, with its cause remained regarded by the mainstream as unknown $(3,4)$. Up to date, all the treatments are mainly targeting the inflammation, using corticosteroids, immunosuppressive agents such as azathioprine or 6-mercaptopurine, anti-inflammatory agents such as 5-aminosalicates, or biologics such as anti-TNF- $\alpha$ antibodies (5-7).

Multiple studies showed that patients or animals with IBD have increased fecal digestive proteases such as trypsin and chymotrypsin (8-10). Furthermore, the serine proteases inhibitors (e.g., Bowman-Birk protease inhibitor, BBI) are important anti-inflammatory agents for various inflammations (e.g., skin rosacea, multiple sclerosis) and autoimmune diseases (11-13). Especially, the therapeutic effect of BBI on IBD patients or experimental animal colitis was confirmed $(14,15)$. The digestive enzymes located in the GI tract are the potential and vital therapeutic targets for IBD treatment accordingly $(16,17)$.

As an important endogenous substance largely distributed in GI tract, the unconjugated bilirubin (UCB) from heme metabolism by the heme oxygenase-1 (HO-1) is an effective antioxidant (18). Our recent studies using bile duct ligated rats confirmed the critical role of unconjugated bilirubin in inactivation of digestive proteases and gut protection $(19,20)$. Whereas, the specific effects of UCB on the inflammation in colitis, and the modifications of digestive proteases levels are still unrevealed. Therefore, we designed a UCB treatment study on an experimental colitis rats model to confirm the effect of UCB 
Table I. Criteria for the assessment of macroscopic colonic damage scores.

\begin{tabular}{lcl}
\hline Criteria & Score & \multicolumn{1}{c}{ Appearance } \\
\hline Ulceration and inflammation & 0 & Normal, no damage \\
& 1 & Focal hyperemia, no ulcers \\
Ulcer without significant inflammation & Ulcer with significant inflammation at one site \\
& 3 & Two or more major sites of ulceration/inflammation, or major \\
& $4-5$ & sites extending $>1$ cm along the length of colon \\
Major damage extending $>2$ cm along the length of colon, and \\
the score is increased by one point for each additional \\
centimeter of damage \\
Adhesions
\end{tabular}

on colitis management and the levels of digestive proteases in feces.

\section{Materials and methods}

Animals. Male Sprague-Dawley (SD) rats (weight $\sim 180 \mathrm{~g}$ ) were purchased from the Experimental Animal Center of the Second Affiliated Hospital of Harbin Medical University. The study was approved by the Animal Care and Use Committee of the Harbin Medical University.

Drugs and reagents. Trinitrobenzenesulfonic acid (TNBS) and unconjugated bilirubin (UCB) were purchased from Sigma-Aldrich (St. Louis, USA). ELISA kits for trypsin, chymotrypsin, TNF- $\alpha$, IL-1 $\beta$ and MPO were obtained from Beijing Propbs Biotechnology Co., Ltd. (Beijing, China).

Induction of colitis and treatment with UCB. TNBS-induced colitis was established as described previously (21). SD rats were randomly divided into three groups: The normal control group (Control group), the TNBS model group (TNBS group) and TNBS model rats treated with UCB group (TNBS + UCB group). After a $24 \mathrm{~h}$ fasting, the animals were slightly anesthetized with amobarbital sodium $(25 \mathrm{mg} / \mathrm{K}$, i.p.), and then a medical-grade polyurethane cannula was inserted into the anus with the tip positioned about $8 \mathrm{~cm}$ proximally to the anus. TNBS group received colonic instillation of $1 \mathrm{ml}$ of $50 \%$ ethanol in saline containing $25 \mathrm{mg}$ TNBS, while the control group received $1 \mathrm{ml}$ saline $(22,23)$. After colonic instillation, the UCB treatment group received an intra-gastric gavages of $3.5 \mathrm{ml} \mathrm{UCB}(40 \mu \mathrm{M}$, UCB is dissolved in $0.4 \%$ dimethyl sulfoxide at concentrations up to $40 \mu \mathrm{M})(19,23)$, while the Control and TNBS groups received equal volume of saline solution. All animals were recorded daily for body weight and total feces were collected daily and stored at $-4^{\circ} \mathrm{C}(24)$. On day 1,3 and 7 after UCB treatment, rats were sacrificed and colon about $8 \mathrm{~cm}$ above the anus was harvested and stored for further analysis (19,22-24).

Assessment of colonic damage. Colonic damage was assessed by both Macroscopic Damage Scores (MDS) as shown in
Table II. Criteria for the assessment of microscopic colonic inflammation scores.

\begin{tabular}{lc}
\hline Criteria & Score \\
\hline Inflammatory cell infiltrate & $0-3$ \\
Gut wall layers infiltrated & $0-3$ \\
Loss of mucosal architecture & $0-3$ \\
Edema & $0-1$ \\
Max score & 10 \\
\hline
\end{tabular}

Table I $(25,26)$, and histological inflammation scores using Hematoxylin and eosin (H\&E) staining (19), based on the following parameters (Table II): Inflammatory cell infiltrate, loss of mucosal architecture, gut wall layers infiltrated, and edema $(19,27)$.

Assay of trypsin and chymotrypsin in feces, and TNF- $\alpha, I L-1 \beta$ and myeloperoxidase (MPO) in colonic tissue. The concentrations of trypsin and chymotrypsin in feces, TNF- $\alpha$, IL-1 $\beta$ and myeloperoxidase (MPO) in colon tissue, were assessed by ELISA kits, based on the manufacturer's instructions. Results of trypsin, TNF- $\alpha$, IL-1 $\beta$ were expressed as $\mathrm{pg} / \mathrm{mg}$, and chymotrypsin and MPO were expressed as $\mathrm{U} / \mathrm{g}$.

Statistical analysis. Results were expressed as mean \pm SEM. Differences between groups were determined by one-way ANOVA with LSD or Tamhane multiple comparisons post hoc tests, using SPSS version 19.0 (IBM SPSS, Armonk, NY, USA). The correlations were assessed using linear fit in Origin-Pro8 (OriginLab Corporation, Northampton, MA, USA). $\mathrm{P}<0.05$ was considered to indicate a statistically significant difference.

\section{Results}

UCB alleviates loss of body weight in TNBS-treated rats. The body weight of rats was measured once daily for 7 days, and the body weight change relative to pre-treatment of rats was 
Table III. Correlation analysis of digestive proteases and inflammatory markers.

Digestive enzymes

Statistics of linear fit

\begin{tabular}{|c|c|c|c|c|c|c|}
\hline \multirow{2}{*}{$\frac{\text { Statistics of linear fit }}{\text { Inflammatory markers }}$} & \multicolumn{3}{|c|}{ Chymotrypsin (U/g) } & \multicolumn{3}{|c|}{ Trypsin (pg/mg) } \\
\hline & Slope & R-Square & P-value & Slope & R-Square & P-value \\
\hline MPO (U/g) & 1.704 & 0.775 & 0.016 & 0.604 & 0.839 & $3.241 \mathrm{E}-4$ \\
\hline TNF- $\alpha(\mathrm{pg} / \mathrm{mg})$ & 0.455 & 0.749 & 0.007 & 0.212 & 0.916 & $3.276 \mathrm{E}-5$ \\
\hline IL-1 $\beta(\mathrm{pg} / \mathrm{mg})$ & 0.112 & 0.750 & 0.007 & 0.033 & 0.823 & 0.001 \\
\hline
\end{tabular}

Pearson's correlation coefficient was used to correlate the digestive enzymes and inflammatory markers. The correlation significance was considered when $\mathrm{P}$ values were lower than 0.05 .

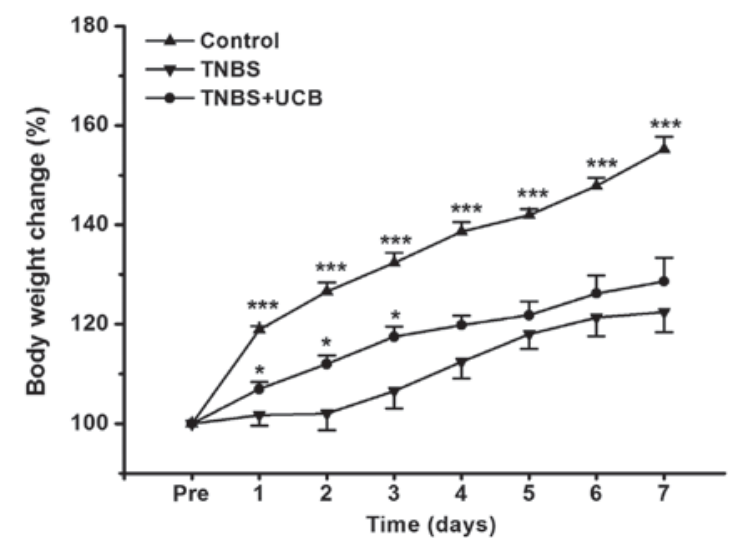

Figure 1. Change of body weight. The body weight change relative to pre-treatment was plotted and the data was expressed as mean \pm SEM $(n=5$ in each group). ${ }^{*} \mathrm{P}<0.05,{ }^{* * *} \mathrm{P}<0.001$ vs. TNBS group.

calculated. From our data (Fig. 1), TNBS caused dramatic reduction in body weight gain, while UCB treatment significantly alleviated this body weight loss from day 1 to day $3(\mathrm{P}<0.05)$.

Effects of UCB on macroscopic and histological pathological changes of rats with TNBS-induced colitis. Meanwhile, TNBS caused momentous damage of colonic tissues (Fig. 2A). Furthermore, the MDS of TNBS-treated rats were significant higher than control rats $(\mathrm{P}<0.001$ from day 1 to day 7$)$, whereas the MDS of UCB treated rats was significantly lower compared to TNBS alone ( $\mathrm{P}<0.01$ at day 3 and 7$)$ (Fig. 2B and Table I). While there was no significant difference at day 1 and day 7 , our data demonstrated significant ameliorating effect by UCB treatment at day 3 with histological staining (Fig. 3A) and microscopic colonic inflammation scores (Fig. 3B, $\mathrm{P}<0.001$ ).

Effects of UCB on TNBS-induced increases of pro-inflammatory cytokines in the colon tissue. Same as reported by others (28), the results of our experiment showed TNBS caused significant increases in TNF- $\alpha$, IL-1 $\beta$ and MPO in the colon, while treatment with UCB alleviates these changes (Fig. 4A-C).

Changes offecal digestive proteases. As previous studies showed that the fecal digestive proteases (trypsin and chymotrypsin) were increased in IBD $(8,10,29)$. Therefore, we measured the

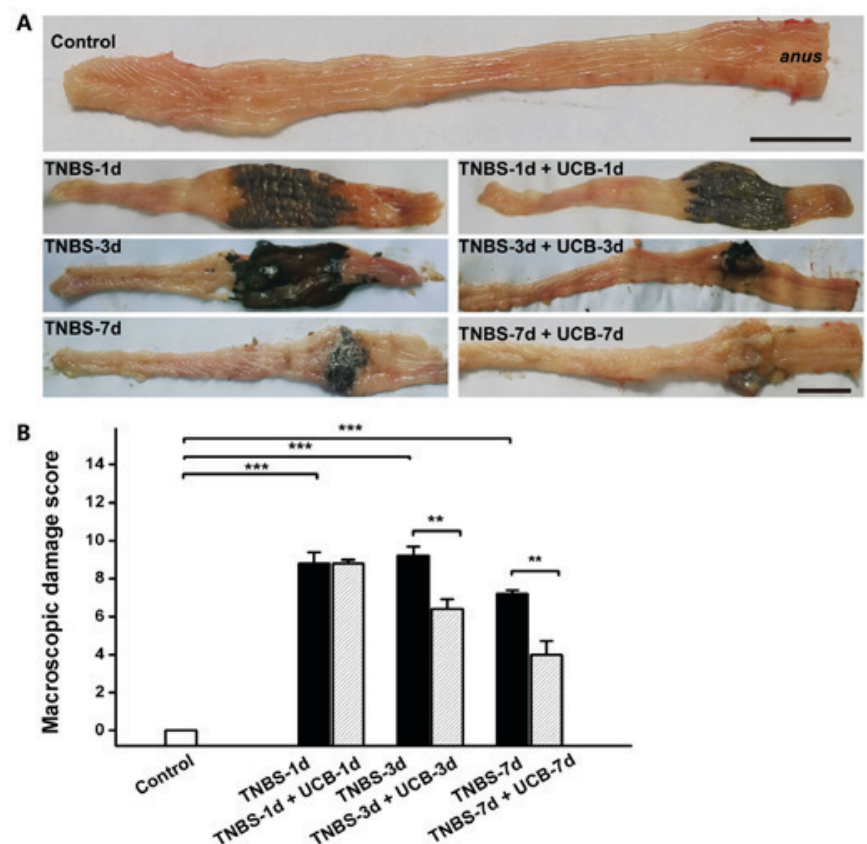

Figure 2. Macroscopic Damage of the colon. (A) Representative images of macroscopic damage of the colon. Scale bars, $2 \mathrm{~cm}, \mathrm{n}=4-6$ in each group. (B) Macroscopic Damage Scores (MDS). Data are expressed as mean \pm SEM and results were analyzed using Student's t-test, $n=4-6$ in each group. ${ }^{* *} \mathrm{P}<0.01,{ }^{* * *} \mathrm{P}<0.001$.

fecal trypsin and chymotrypsin levels of the different groups of rats. Our results demonstrated that both trypsin and chymotrypsin were significant increased from day 1 to 7 after TNBS treatment (Fig. 5), however, the UCB treatment significantly reduced the levels of trypsin and chymotrypsin $(\mathrm{P}<0.01$ on day 3). To explore the relationship among changes of digestive proteases in gut lumen and pro-inflammatory cytokines in colon tissue, we further conducted a correlation analysis among these parameters using the data collected on day 3. It showed positive significant correlations (Pearson's correlation coefficient, slop $>0, \mathrm{P}<0.05$ ) among these parameters (Table III).

\section{Discussion}

UCB is previously known as a toxic endogenous substance on nervous system in high concentrations, but also a pivotal 
A

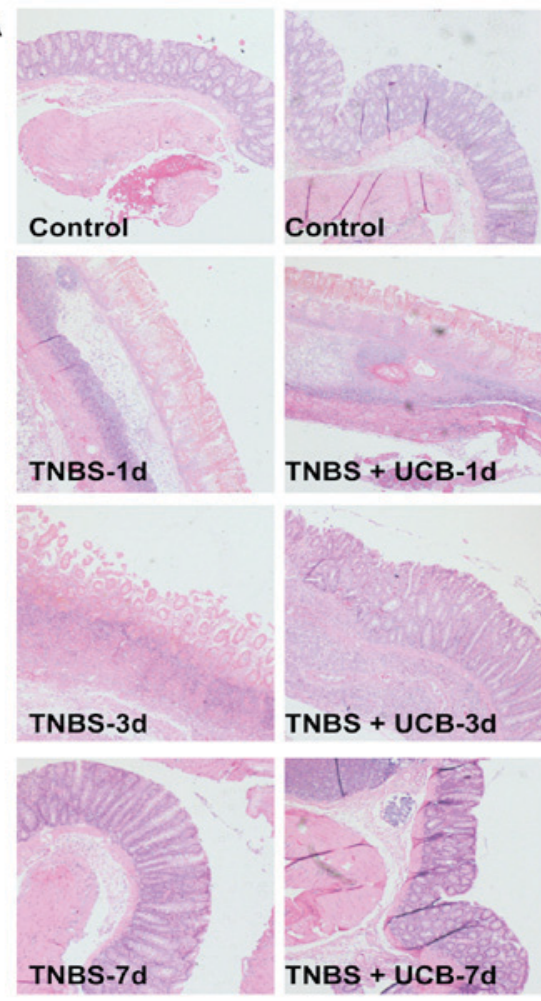

B

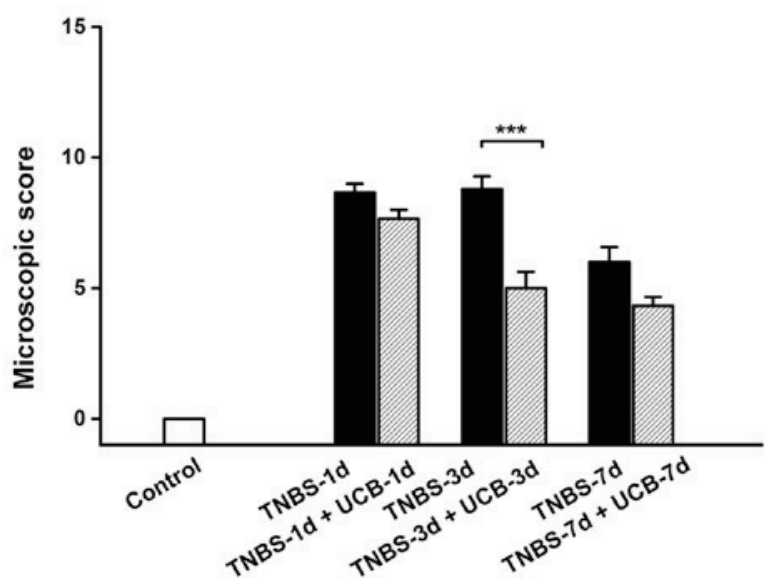

Figure 3. Representative images of histological changes of the colon (A) H\&E staining (magnification, x40). (B) Microscopic colonic inflammation scores. Data are expressed as mean \pm SEM and results were analyzed using Student's $t$-test, $n=3-6$ in each group. ${ }^{* * * *} \mathrm{P}<0.001$.
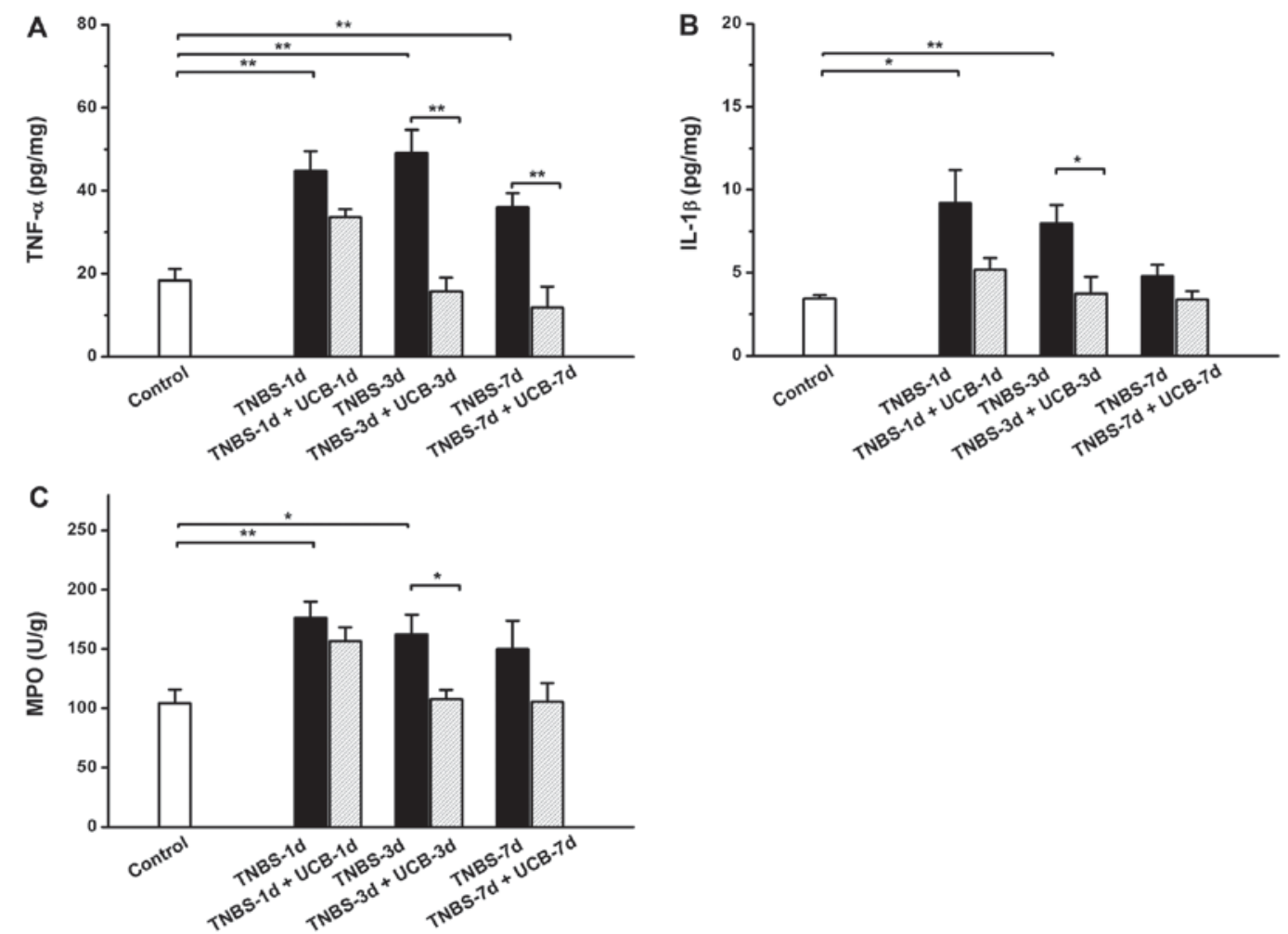

Figure 4. Changes of (A) TNF- $\alpha$, (B) IL-1 $\beta$ and (C) MPO in colonic tissues. Data points are expressed as mean \pm SEM ( $n=5$ in each group). ${ }^{*} \mathrm{P}<0.05,{ }^{* *} \mathrm{P}<0.01$ as analyzed by the Student's t-test.

antioxidant in low concentrations (18), that plays an important potential protection role in vascular endothelial function, experimental murine colitis (30-32), and other disorders including non-alcoholic steatohepatitis and advanced fibrosis (30). Moreover, as a key upstream modulator for endogenous biliverdin generation, $\mathrm{HO}-1$ has been proved with various protective 

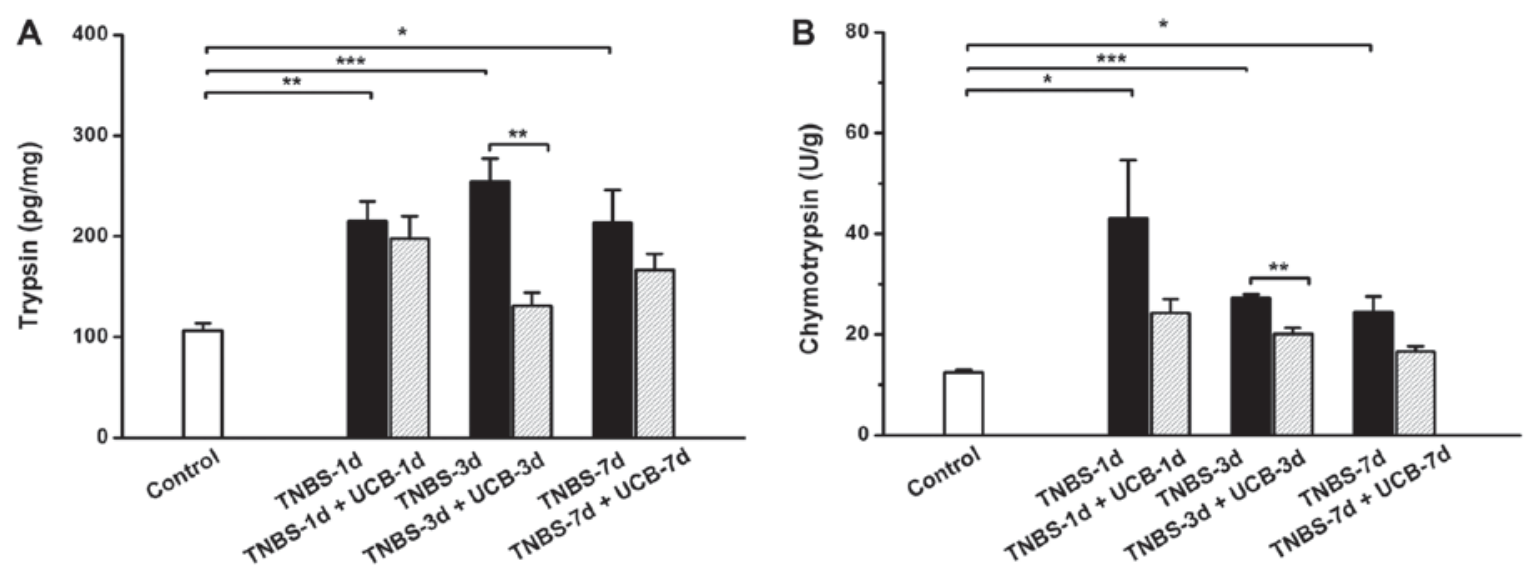

Figure 5. Changes of (A) trypsin and (B) chymotrypsin. Data are expressed as mean \pm SEM ( $\mathrm{n}=5$ in each group). ${ }^{*} \mathrm{P}<0.05,{ }^{* *} \mathrm{P}<0.01,{ }^{* * *} \mathrm{P}<0.001$ as analyzed by Student's t-test.

effects on atherosclerosis $(30,33)$, immuno-related inflammation (33), and also experimental murine colitis mediated by UCB (31,34). However, the anti-inflammatory mechanism of HO-1/UCB axis are still unclear, and the role of UCB on inflammatory bowel diseases with anti-inflammatory property is attractive.

In our previous studies, we have observed increased activities of fecal trypsin and chymotrypsin in animals with bile duct ligation (BDL) $(19,20)$. From results above, UCB administrated ameliorates the tissue damage and inflammation in the gastrointestinal tract of TNBS-induced colitis. Interestingly, the levels of trypsin and chymotrypsin in feces of TNBS group were both significantly increased, and ameliorated under the UCB treatment, while did not change after administration in normal control rats (data not shown). Moreover, significant positive correlations were identified by the linear fitting analysis results of digestive enzymes (trypsin and chymotrypsin) and inflammatory markers levels (MPO, TNF- $\alpha$ and IL-1 $\beta$ ). In addition, proteases are important in inflammation process via protease activated receptors in various tissues $(11,12,29)$, and the anti-inflammatory roles of digestive enzymes inhibitors or serine protease inhibitors for trypsin and chymotrypsin has been reported (17). Therefore, the digestive proteases could be the key mediators and targets for the anti-inflammatory effects of UCB, and the proteases reduction by UCB would be one of the important therapeutic pathway for the treatment of inflammatory diseases like colitis. Notably, the limitations of this study are that the detail mechanisms for direct anti-inflammatory effects or digestive proteases inactivation dependent anti-inflammatory effects of UCB on colitis are still unrevealed, while our results in this study have demonstrated that the UCB treatment ameliorates the inflammation and digestive proteases increase in the gastrointestinal tract of TNBS-induced colitis.

\section{Acknowledgements}

We are grateful to thank Kangkang Zhou and Guojing Shi for excellent laboratory assistance, and Fu-Lai Chen for the histological staining of colonic tissues. This study was supported by the National Natural Science Foundation of China (no. 30973596).

\section{References}

1. Bouma G and Strober W: The immunological and genetic basis of inflammatory bowel disease. Nat Rev Immunol 3: 521-533, 2003.

2. Mokry M, Middendorp S, Wiegerinck CL, Witte M, Teunissen H, Meddens CA, Cuppen E, Clevers H and Nieuwenhuis EE: Many inflammatory bowel disease risk loci include regions that regulate gene expression in immune cells and the intestinal epithelium. Gastroenterology 146: 1040-1047, 2014.

3. Cosnes J, Gower-Rousseau C, Seksik P and Cortot A: Epidemiology and natural history of inflammatory bowel diseases. Gastroenterology 140: 1785-1794, 2011.

4. Kirsner JB: The historical basis of the idiopathic inflammatory bowel diseases. Inflammatory Bowel Dis 1: 2-26, 1995.

5. Pithadia AB and Jain S: Treatment of inflammatory bowel disease (IBD). Pharmacol Rep 63: 629-642, 2011.

6. Rietdijk ST and D'Haens GR: Recent developments in the treatment of inflammatory bowel disease. J Dig Dis 14: 282-287, 2013.

7. Sohrabpour AA, Malekzadeh R and Keshavarzian A: Current therapeutic approaches in inflammatory bowel disease. Curr Pharm Des 16: 3668-3683, 2010.

8. van de Merwe JP and Mol GJ: Levels of Trypsin and alpha-chymotrypsin in feces from patients with Crohn's disease. Digestion 24: $1-4,1982$.

9. Maeda S, Ohno K, Uchida K, Igarashi H, Goto-Koshino Y, Fujino $\mathrm{Y}$ and Tsujimoto H: Intestinal protease-activated receptor-2 and fecal serine protease activity are increased in canine inflammatory bowel disease and may contribute to intestinal cytokine expression. J Vet Med Sci 76: 1119-1127, 2014.

10. Midtvedt T, Zabarovsky E, Norin E, Bark J, Gizatullin R, Kashuba V, Ljungqvist O, Zabarovska V, Möllby R and Ernberg I: Increase of faecal tryptic activity relates to changes in the intestinal microbiome: Analysis of Crohn's disease with a multidisciplinary platform. PLoS One 8: e66074, 2013.

11. Safavi F and Rostami A: Role of serine proteases in inflammation: Bowman-Birk protease inhibitor (BBI) as a potential therapy for autoimmune diseases. Exp Mol Pathol 93: 428-433, 2012.

12. Yamasaki K, Di Nardo A, Bardan A, Murakami M, Ohtake T, Coda A, Dorschner RA, Bonnart C, Descargues P, Hovnanian A, et al: Increased serine protease activity and cathelicidin promotes skin inflammation in rosacea. Nat Med 13: 975-980, 2007.

13. Gran B, Tabibzadeh N, Martin A, Ventura ES, Ware JH, Zhang GX, Parr JL, Kennedy AR and Rostami AM: The protease inhibitor, Bowman-Birk Inhibitor, suppresses experimental autoimmune encephalomyelitis: A potential oral therapy for multiple sclerosis. Mult Scler 12: 688-697, 2006.

14. Lichtenstein GR, Deren JJ, Katz S, Lewis JD, Kennedy AR and Ware JH: Bowman-Birk inhibitor concentrate: A novel therapeutic agent for patients with active ulcerative colitis. Dig Dis Sci 53: 175-180, 2008.

15. Ware JH, Wan XS, Newberne P and Kennedy AR: Bowman-Birk inhibitor concentrate reduces colon inflammation in mice with dextran sulfate sodium-induced ulcerative colitis. Dig Dis Sci 44: 986-990, 1999. 
16. Qin XF: Impaired inactivation of digestive proteases by deconjugated bilirubin: The possible mechanism for inflammatory bowel disease. Med Hypotheses 59: 159-163, 2002.

17. Bermúdez-Humarán LG, Motta JP, Aubry C, Kharrat P, Rous-Martin L, Sallenave JM, Deraison C, Vergnolle N and Langella P: Serine protease inhibitors protect better than IL-10 and TGF- $\beta$ anti-inflammatory cytokines against mouse colitis when delivered by recombinant lactococci. Microb Cell Fact 14: 26, 2015.

18. Stocker R, Yamamoto Y, McDonagh AF, Glazer AN and Ames BN: Bilirubin is an antioxidant of possible physiological importance. Science 235: 1043-1046, 1987.

19. Zhou K, Jiang M, Liu Y, Qu Y, Shi G, Yang X, Qin X and Wang X: Effect of bile pigments on the compromised gut barrier function in a rat model of bile duct ligation. PLoS One 9: e98905, 2014.

20. Zhou K, Jiang M, Qin X and Wang X: Role of bilirubin in digestive proteases inactivation in the lower intestine. Dig Liver Dis 47: 438-439, 2015.

21. Morris GP, Beck PL, Herridge MS, Depew WT, Szewczuk MR and Wallace JL: Hapten-induced model of chronic inflammation and ulceration in the rat colon. Gastroenterology 96: 795-803, 1989.

22. Szalai Z, Szász A, Nagy I, Puskás LG, Kupai K, Király A, Berkó AM, Pósa A, Strifler G, Baráth Z, et al: Anti-inflammatory effect of recreational exercise in TNBS-induced colitis in rats: Role of NOS/HO/MPO system. Oxid Med Cell Longev 2014: 925981, 2014.

23. Zhou K, Jiang M, Qin X and Wang X: Role of bilirubin in digestive proteases inactivation in the lower intestine. Dig Liver Dis 47: 438-439, 2015

24. Gilani GS and Sepehr E: Protein digestibility and quality in products containing antinutritional factors are adversely affected by old age in rats. J Nutr 133: 220-225, 2003.

25. Camuesco D, Peran L, Comalada M, Nieto A, Di Stasi LC, Rodriguez-Cabezas ME, Concha A, Zarzuelo A and Galvez J: Preventative effects of lactulose in the trinitrobenzenesulphonic acid model of rat colitis. Inflamm Bowel Dis 11: 265-271, 2005.
26. Aube AC, Cherbut C, Barbier M, Xing JH, Roze C and Galmiche JP: Altered myoelectrical activity in noninflamed ileum of rats with colitis induced by trinitrobenzene sulphonic acid. Neurogastroenterol Motil 11: 55-62, 1999.

27. Ruyssers NE, De Winter BY, De Man JG, Loukas A, Pearson MS, Weinstock JV, Van den Bossche RM, Martinet W, Pelckmans PA and Moreels TG: Therapeutic potential of helminth soluble proteins in TNBS-induced colitis in mice. Inflamm Bowel Dis 15: 491-500, 2009.

28. Kapitulnik J: Bilirubin: An endogenous product of heme degradation with both cytotoxic and cytoprotective properties. Mol Pharmacol 66: 773-779, 2004.

29. Maeda S, Maeda S, Ohno K, Kaji N, Hori M, Fujino Y and Tsujimoto H: Protease-activated receptor-2 induces proinflammatory cytokine and chemokine gene expression in canine keratinocytes. Vet Immunol Immunopathol 153: 17-25, 2013.

30. Liu J, Wang L, Tian XY, Liu L, Wong WT, Zhang Y, Han QB, Ho HM, Wang N, Wong SL, et al: Unconjugated bilirubin mediates heme oxygenase-1-induced vascular benefits in diabetic mice. Diabetes 64: 1564-1575, 2015.

31. Berberat PO,A-Rahim YI, Yamashita K, Warny MM, Csizmadia E, Robson SC and Bach FH: Heme oxygenase-1-generated biliverdin ameliorates experimental murine colitis. Inflamm Bowel Dis 11: 350-359, 2005.

32. Kawamura K, Ishikawa K, Wada Y, Kimura S, Matsumoto H, Kohro T, Itabe H, Kodama $\mathrm{T}$ and Maruyama Y: Bilirubin from heme oxygenase-1 attenuates vascular endothelial activation and dysfunction. Arterioscler Thromb Vasc Biol 25: 155-160, 2005.

33. Lee TS and Chau LY: Heme oxygenase-1 mediates the anti-inflammatory effect of interleukin-10 in mice. Nat Med 8: 240-246, 2002.

34. Varga C, Laszlo F, Fritz P, Cavicchi M, Lamarque D, Horvath K, Posa A, Berko A and Whittle BJ: Modulation by heme and zinc protoporphyrin of colonic heme oxygenase-1 and experimental inflammatory bowel disease in the rat. Eur J Pharmacol 561: $164-171,2007$. 